\title{
PLCC with Sensors and Microcontroller Networking For Power Management
}

\author{
Nitesh Kumar Jangir
}

\begin{abstract}
This paper gives information of power handling by means of communication system and using their net for auto and manual power management at very cheap cost. In this project we use existing network of power line for the data communication by using a network of microcontrollers. The microcontroller uses sensors for intake values from atmosphere than control electric and electronics equipments according to instructions. Project proposes to use Power lines as a medium to carry control data to control a load.
\end{abstract}

Index Terms-Networking; power lines; protocols; sensors; trapping.

\section{INTRODUCTION}

In present age of information technology present focus is on both creation as well as dispersion of information. In order to able to reach the end users for provision of information, the popular means are include telephone wires, Ethernet cables, and fiber optics, wireless and satellite technologies. Each of these has limitation of cost and availability to reach maximum number of users.

PLCC is power line carrier communication system in which we use all type of power carrying lines for example high tension, low power for telephonic communication in my technique, we use this technology for the power controlling and management. Advantage of use of power lines as a data transmission medium is that each home and building already equipped with power lines. Using this technology we can auto -control the power consumption and also see on the screen amount of power consumed. As we know that there is some technologies already in market say SCADA (supervision control and data acquisition), but these technologies are very expensive as respect to the technology. This has a combination of microcontroller with sensors and use power line as a medium to take data and communication. We can say that it is a microcontroller networking with sensors for real time data analysis and logics for real time controlling. The most important thing about this is that this technique is very cost effective. The potential of power line as a powerful medium to able to deliver not only the electrical and control signals as well as high speed full duplex data transmission. Now we have artificially intelligent systems and that are stronger than humans, that can venture places where people cannot go (Such as Mars), that are smarter than people in certain cases (for example in chess), and so on. This uses an algorithm by using this algorithm fastest communication between micro-

Manuscript received Feburary 20, 2012; revised March 23, 2012.

N. K. Jangir is with the Electro System Associates, Bangalore, India (email: nitujangir@yahoo.com). controller units can be possible and this increases speed of operation and response time of the system. There is one problem arrive in tests that during the transmission of data interference occurs and this leads to the les signal to noise ratio.

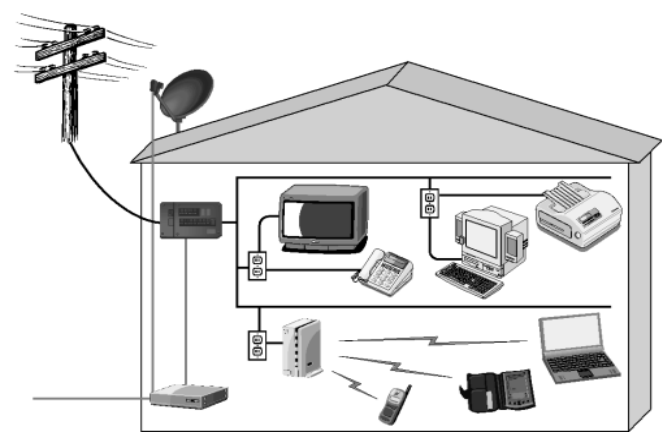

Fig. 1. Power Line connected to home appliances

We are no longer truly surprised when machine artifacts Outperform humans in new domains. So, this system more errorless than a human kind. In microcontroller networking we use any microcontroller that can be 8 to 32 bit as per as systems coverage area and speed and error tolerance. Selection of microcontroller also depends upon the number of sensors, more number of sensors yields to higher level of microcontroller unit.

\section{SYSTEM DESIGN}

The basic device is microcontroller and its peripherals such as RS232 and sensors etc. Power line carrier communication (PLCC) is mainly used for telecommunication, tele-protection and tele-monitoring between electrical substations through power lines at high voltages, such as $110 \mathrm{kV}, 220 \mathrm{kV}$, and $400 \mathrm{kV}$. PLCC integrates the transmission of communication signal and $50 / 60 \mathrm{~Hz}$ power signal through the same electric power cable. The major benefit is the union of two important applications in a single system is that it is more economical than the other present technologies that we have. In system design main task is to select sensor and microcontroller.

\section{A. Line Trap}

It is also called "Wave trap". It is connected in series with the power (transmission) line. It blocks the high frequency carrier waves $(24 \mathrm{kHz}$ to $500 \mathrm{kHz})$ and let power waves $(50$ $\mathrm{Hz}-60 \mathrm{~Hz}$ ) to pass through. It is basically an inductor of rating in milli henrys [2]

\section{B. Coupling Capacitor}

It provides low impedance path for carrier energy to HV line and blocks the power frequency circuit by being a high impedance path. 


\section{Line Matching Unit}

LMU (Line matching unit) is a composite unit consisting of Drain Coil, Isolation transformer with Lightning Arrester on its both the sides, a Tuning Device and an earth switch. Tuning Device is the combination of R-L-C circuits which act as filter circuit. LMU is also known as Coupling Device. Together with coupling capacitor, LMU serves the purpose of connecting effectively the Audio/Radio frequency signals to either transmission line or PLC terminal and protection of the PLCC unit from the overvoltage caused due to transients on power system[2].

\section{Digital Power Line Carrier}

A power line carrier using a power line as transmission media needs to change its transmission system from analog to digital to address rapid diffusion of IP devices and digital telecommunication devices. With this view, digital power line carrier (DPLC) was developed featuring several technological measures which enable digital transmission via power lines and performed a field evaluation test. As a result, DPLC has the required quality of bit error rate characteristics and transmission ability such as transmitting information from monitored electric-supply stations and images [4].

We are using microcontroller and electronic relay as a router, for the generated power. In this Method can say that this is an embedded system for the routing of power. These routers connected to the every node of the circuit. Generally 2or 4 on each floor. These all are connected with the central unit (microcontroller) that have real time operating system.

In practical tests atmel's ATMEGA16 microcontroller is used. This is 8 bit microcontroller work on clock frequency range 0 to $16 \mathrm{MHz}$ and needs supply voltage $4.5-5.5 \mathrm{~V}$

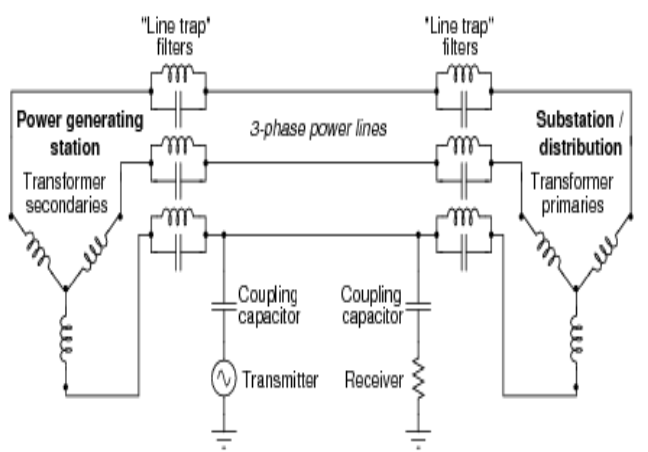

Fig. 2. Schematic of PLCC

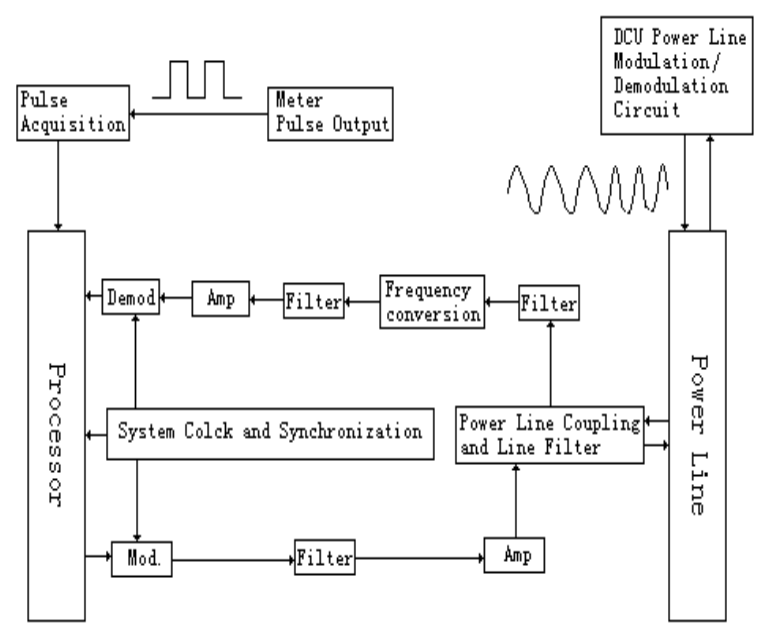

Fig. 3. System design [4]
All the microcontrollers are connected to each other by sending a DataStream. This system uses any of existing shortest path algorithm for the routing of power, it will give tremendous result. We also connect this embedded system to the sensors that are used for the automated lightning, heating, ventilating , air conditioning(HVACs) ,security climate control etc. this energy management solution also can provide capability to power down the devices when they are not in use especially the devices that uses more power and schedule down times for the maximum efficiency . add to this a communication system for the monitor feedback from all electrical loads.

Some examples of microcontrollers for this are AT89C52-24JC, AT89C52 is a 44-Pin work on $24 \mathrm{MHz}$ frequency having memory of $8 \mathrm{~kb} 8$-bit Microcontroller (PLCC).This microcontroller is specially designed for the

\section{COMMONLY USED SENSORS}

A network of sensors is connected to microcontroller. Number of sensors is depends on type of microcontroller unit we use. Generally 8 bit microcontroller can handle 8 to 10 sensors at a time. According to the location where this system has to be install, number and types of sensors can be choose. These sensors provide real-time values of change in environment.

\section{A. $L D R$}

This is sensor that has a resistance that varies according to the amount of visible light that falls on it. This is one the most popular sensor and easily available. This is easily connectable to microcontroller and also very cheap. A close up of an LDR is shown below:
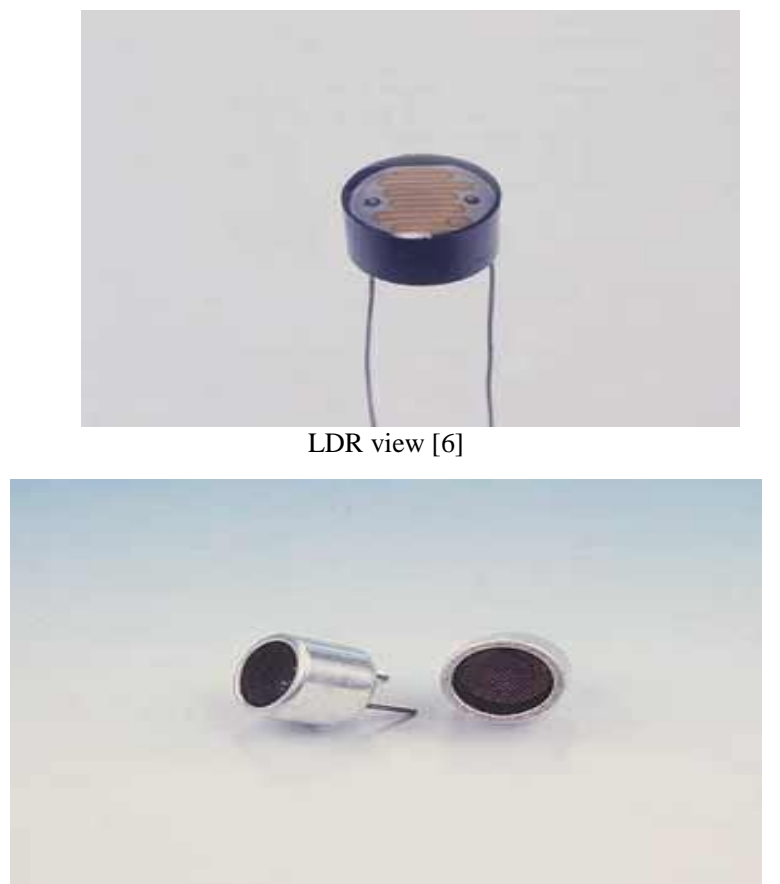

Fig. 4. Ultrasonic view [6]

The light falling on the brown zigzag lines on the sensor causes the resistance of the device to fall. This is known as a negative co-efficient. There are some LDRs that work in the opposite way i.e. their resistance increases with light (called positive co-efficient)[6]. Now, in order to use this 
device in a simple circuit, all we need to do is put a voltage across it and measure the current flowing through it. However, measuring current can be a little tricky. So, put another resistor in series, and measure the voltage across the LDR. This makes us a potential divider, and the voltage across the LDR is proportional to the current. The diagrams below show the concept. Here, the current is directly proportional to the resistance of the LDR.A much easier way is to put a second resistor in series with the LDR and measure the voltage across the LDR (light detecting register).To take the extreme cases, system can therefore detect if light is present or not, just by simply detecting if output have voltage or not. For use two sensors like those on some robots then we can also detect which direction the light is coming from simply by seeing which sensor has the stronger voltage. It plays important role in system design because by using this sensor we got electrical values according to change in the intensity of light in atmosphere and by using output of this microcontroller change intensity of light source. This light source is LED bulbs.

\section{B. Ultrasonic}

These sensors emit a very high frequency sound. In fact it is so high, that we can't hear them. A picture of ultrasonic sensors is shown in Fig 4.Two sensors work in unison, one as the transmitter and one as the receiver (because of this one microcontroller has four of them) below: The transmitter typically sends out a constant beam of sound at a frequency of $40 \mathrm{KHz}$ (note that the human hearing barely goes above $17 \mathrm{KHz}$ ). The receiver detects any sounds coming in and gives us a voltage out. So, what happens is the transmitter sends out a signal. If there isn't an object in front of it, then the sound wave will carry on (note there is a limit to the distance here), If, and only if, there is an object in the way, the sound waves will bounce back along the same path, and so be picked up by our receiver. Now, and this is the clever bit, if we can note the time between transmitting the sound, and when we receive it, then we can work out the distance of the object from our sensors. This, by the way is how parking detectors work on some of the newer cars [6].

\section{Light Sensor}

The principle is very simple. It consists of just two components. The first is an Infra-Red (IR) transmitter (usually an LED), while the second is an Infra-Red receiver (usually a transistor). IR is transmitted out of the sensor unit. If the IR is reflected back, it is picked up by the IR receiver transistor. IR is basically heat (the heat from the sun is predominantly in the IR part of the spectrum). Black, as probably known, absorbs heat, which is why it is best not to wear black in the summer months. If black absorbs heat, then it also absorbs IR. And this is the principle. While the sensor is over a black line, no IR is reflected back to the receiver. If the sensor strays away from the line, then IR is reflected back. a black color. For best results the black color is placed on a white background, which will give the extreme two cases - white reflects IR. Method uses these sensors in detection of color as per system requirement.

\section{D. $L M 35$}

The LM35 series are precision integrated-circuit temperature sensors, whose output voltage is linearly proportional to the Celsius (Centigrade) temperature. The LM35 thus has an advantage over linear temperature sensors calibrated in ${ }^{\circ}$ Kelvin, as the user is not required to subtract a large constant voltage from its output to obtain convenient Centigrade scaling. The LM35 does not require any external calibration or trimming to provide typical accuracies of $\pm 1 / 4{ }^{\circ} \mathrm{C}$ at room temperature and $\pm 3 / 4^{\circ} \mathrm{C}$ over a full -55 to $+150^{\circ} \mathrm{C}$ temperature range. Low cost is assured by trimming and calibration at the wafer level. The LM35's low output impedance, linear output, and precise inherent calibration make interfacing to readout or control circuitry especially easy. It can be used with single power supplies, or with plus and minus supplies. As it draws only $60 \mu \mathrm{A}$ from its supply, it has very low self-heating, less than $0.1^{\circ} \mathrm{C}$ in still air. The $\mathrm{LM} 35$ is rated to operate over a $-55^{\circ}$ to $+150^{\circ} \mathrm{C}$ temperature range, while the $\mathrm{LM} 35 \mathrm{C}$ is rated for a $-40^{\circ}$ to $+110^{\circ} \mathrm{C}$ range $\left(-10^{\circ}\right.$ with improved accuracy). The LM35 series is available packaged in hermetic TO-46 transistor packages, while the LM35C, LM35CA, and LM35D are also available in the plastic TO-92 transistor package [6].. This already comes with the calibration to the microcontrollers and computers. it give information in change in temperature of atmosphere \& according to this power to cooling and heating alliance is change.

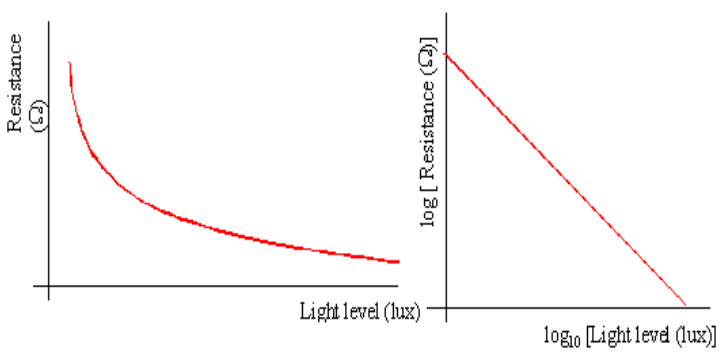

Fig. 5(a). Graph of change in resistance in LDR [5]

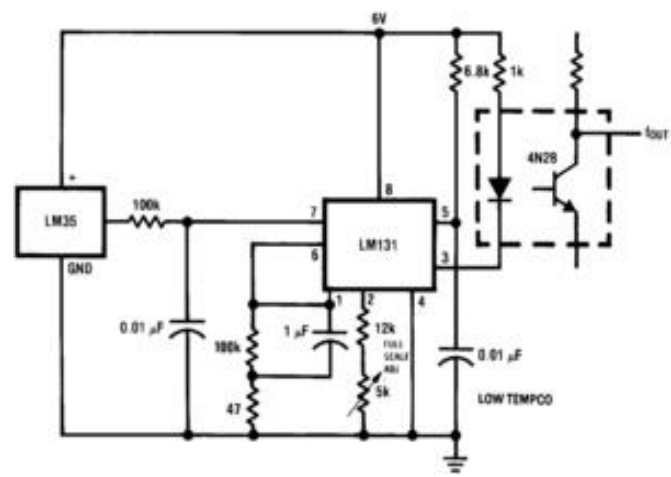

Fig: 5(b) circuit diagram of LM35 [6]

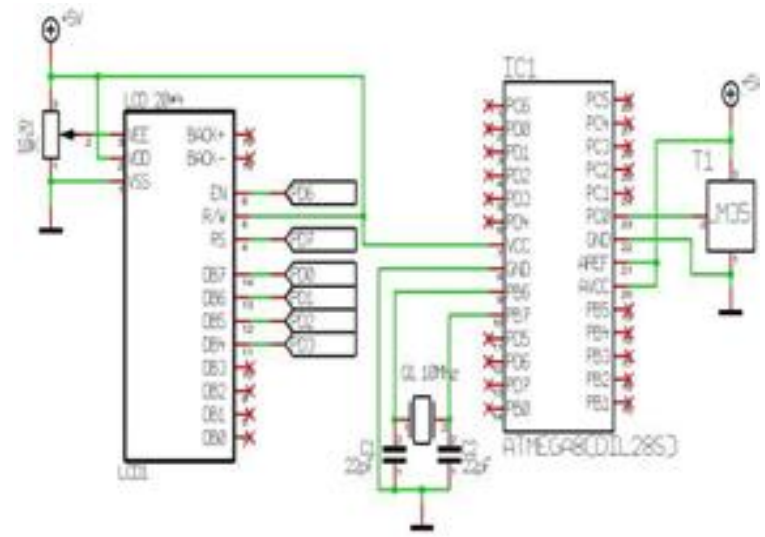

Fig. 5(c). LM35with microcontroller [7] 
In above Fig.5 (b) and 5(c) working, calibration and internal circuitry of this sensor LM35[6].

\section{THE OVERALL SySTEM CONNECTION}

Now connect all these sensors as manner that these all give a desirable response, for this we use pwm (pulse width modulation) mode of microcontroller. In this method set a reference value for the temp, luminous according to general conditions .In practical test method that is used is set microcontroller units in the different portions of the home or office with sensors. All these microcontrollers communicate to each other by plcc .we can use LCD for the display of information of temperature, moisture and light and also give manual control to user.

\section{CONCLUSION}

In this paper the technology is only simple intrigation of technology, can be useable to help in tackle the most important problem standing in front of human kind that is power. By the experiment that have done in this project give a result that this technology yields to save15-20\% of power saving. User can connect this system to any small size business firm or home or building at very low cost of 1000015000INR

\section{REFERENCES}

[1] atmel atmega16 datashet

[2] T. N. Buti, S. Ogura, N. Rovedo, and K. Tobimatsu, "A New AsymmetricalDesign for Reliability and Performance," IEEE Trans. on Elec.Dev, vol. 38, pp. 1757-1764, 1991.

[3] A. Akturk, N. Goldsman, and G. Metze, "Faster Inverter Switching Obtained with Channel Engineered Asymmetrical Halo Implanted," Semi. Dev. Res. Sym, pp. 118-221, 2001.

[4] A. eHaidine, H. Hrasnica, and R. Lehnert, "Broadband Powerline Communications: Network Design," pp. 110-150, 2000.

[5] Taurus Device Simulater, v2003.06, Synopsys, Inc.

[6] Device home page, http://wwwmtl.mit.edu/Well/, Available online.

[7] M. Stockinger, R. Strasser, R. Plasum, A. Wild, and S. Selberherr, "A Microcontroller and embedded systems," pp. 77-80, 1998.

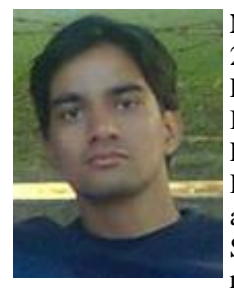

Nitesh Kumar Jangir is born on 23/08/1989 in India. 2007-11 B.E.,Electronics \& Communication Engineering, Rajiv Gandhi Technological University, India. He is working at Electro System Associates Pvt. Ltd., Bangalore, India as Embedded System Engineer. He published papers in international conferences \& filed a patent. His areas of interest is Embedded Systems,sensor network, Medical electronics \& robotics. 